\title{
ESPE/LWPES consensus statement on diabetic ketoacidosis in children and adolescents
}

\author{
D B Dunger, M A Sperling, C L Acerini, D J Bohn, D Daneman, T P A Danne, N S Glaser, R Hanas, \\ R L Hintz, L L Levitsky, M O Savage, R C Tasker, J I Wolfsdorf
}

Diabetic ketoacidosis (DKA) is the leading cause of morbidity and mortality in children with type 1 diabetes mellitus (TIDM). Mortality is predominantly related to the occurrence of cerebral oedema; only a minority of deaths in DKA are attributed to other causes. Cerebral oedema occurs in about $0.3-1 \%$ of all episodes of DKA, and its aetiology, pathophysiology, and ideal method of treatment are poorly understood. There is debate as to whether physicians treating DKA can prevent or predict the occurrence of cerebral oedema, and the appropriate site(s) for children with DKA to be managed. There is agreement that prevention of DKA and reduction of its incidence should be a goal in managing children with diabetes.

See end of article for authors' affiliations

Correspondence to: Correspondence to:
Prof. D B Dunger, University of Cambridge, Department of Paediatrics, Addenbrooke's Hospital, Level 8, Box 116 Cambridge CB2 2QQ, UK; dbd25@cam.ac.uk

Accepted 27 October 2003
$\mathrm{T}$ o explore these issues, the Lawson Wilkins Pediatric Endocrine Society (LWPES) and the European Society for Paediatric Endocrinology (ESPE) convened a panel of expert physicians for a Consensus Conference. The meeting was chaired by Mark A. Sperling, representing LWPES, and David B. Dunger, representing ESPE. The consensus statement was developed with close partnership between the ESPE and LWPES and the International Society for Pediatric and Adolescent Diabetes (ISPAD), all three organisations being represented by members who participated in the writing process. The statement was also endorsed by related organisations; the Juvenile Diabetes Research Foundation International (JDRFI), the World Federation of Pediatric Intensive and Critical Care Societies, the European Society for Pediatric Critical Care, the European Society of Paediatric and Neonatal Intensive Care (ESPNIC), and the Australian Pediatric Endocrine Group (APEG) were represented by invited participants.

Each of the major topics had a presenter and recorder, responsible for review of the literature and for providing evidence based recommendations according to criteria used by the American Diabetes Association (ADA) (see Appendix; levels of evidence are indicated in bold throughout the text). ${ }^{1}$ Type 2 diabetes was not considered. All participants contributed significantly to the development of consensus.

This document summarises the final consensus reached and represents the current "state of the art".
DEFINITION OF DIABETIC KETOACIDOSIS Diabetic ketoacidosis (DKA) is caused by a decrease in effective circulating insulin associated with increases in counter regulatory hormones including glucagon, catecholamines, cortisol, and growth hormone. This leads to increased glucose production by the liver and kidney and impaired peripheral glucose utilisation with resultant hyperglycaemia, and hyperosmolality. Increased lipolysis, with ketone body (beta-hydroxybutyrate, acetoacetate) production causes ketonaemia and metabolic acidosis. Hyperglycaemia and acidosis result in osmotic diuresis, dehydration, and obligate loss of electrolytes. The biochemical criteria for the diagnosis of DKA include hyperglycaemia (blood glucose $>11 \mathrm{mmol} / \mathrm{l}(\sim 200 \mathrm{mg} / \mathrm{dl}))$ with a venous $\mathrm{pH}<7.3$ and/or bicarbonate $<15 \mathrm{mmol} / \mathrm{l}$. There is associated glycosuria, ketonuria, and ketonaemia. Rarely, young or partially treated children as well as pregnant adolescents may present with near normal glucose values ("euglycaemic ketoacidosis"). ${ }^{2}$

DKA is generally categorised by the severity of the acidosis; varying from mild (venous $\mathrm{pH}$ $<7.30$, bicarbonate concentration $<15 \mathrm{mmol} / \mathrm{l}$ ), to moderate $(\mathrm{pH}<7.2$, bicarbonate $<10)$, to severe $(\mathrm{pH}<7.1$, bicarbonate $<5))^{3{ }^{4}}$

\section{FREQUENCY OF DKA}

\section{At disease onset}

There is wide geographic variation in the frequency of DKA at diabetes onset and rates correlate inversely with regional incidence of TIDM. Reported frequencies range between 15\% and $67 \%$ in Europe and North America and may be more common in developing countries (A). ${ }^{56}$ In Canada and Europe, hospitalisation rates for DKA in established and new patients with TIDM have remained stable at about 10 per 100000 children over the past 20 years, but severity may be decreasing $\mathbf{( B )} .^{78}$

DKA at onset of TIDM is more common in younger children $(<4$ years of age), children without a first degree relative with TIDM, and those from families of lower socioeconomic status (A). ${ }^{49}$ High dose glucocorticoids, atypical antipsychotics, diazoxide, and

Abbreviations: DKA, diabetic ketoacidosis; ECF, extracellular fluid; GFR, glomerular filtration rate; ICF, intracellular fluid; ICP, intracranial pressure; TIDM, type 1 diabetes mellitus 
some immunosuppressive drugs have been reported to precipitate DKA in individuals not previously diagnosed with $\operatorname{TIDM}(\mathbf{B}) .^{10} 11$

\section{In children with established TIDM}

The risk of DKA in established TIDM is $1-10 \%$ per patient per year (A). ${ }^{12-15}$ Risk is increased in children with poor metabolic control or previous episodes of DKA; peripubertal and adolescent girls; children with psychiatric disorders, including those with eating disorders; and those with difficult family circumstances, including lower socioeconomic status and lack of appropriate health insurance. ${ }^{16}$ Inappropriate interruption of insulin pump therapy also leads to DKA. ${ }^{12} 14$

Children whose insulin is administered by a responsible adult rarely have episodes of DKA (C), ${ }^{17}$ and $75 \%$ of episodes of DKA beyond diagnosis are probably associated with insulin omission or treatment error. ${ }^{17}{ }^{18}$ The remainder are due to inadequate insulin therapy during intercurrent illness (B). ${ }^{18-20}$

MORBIDITY AND MORTALITY OF DKA IN CHILDREN Reported mortality rates from DKA in national population based studies are reasonably constant: $0.15 \%$ (C) (USA), ${ }^{21}$ $0.18 \%$ (C) (Canada), ${ }^{7} 0.25 \%$ (C) (Canada), ${ }^{22}$ and $0.31 \%$ (B) (UK). ${ }^{23}$ In places with less developed medical facilities, the risk of dying from DKA is greater, and children may die before receiving treatment. ${ }^{23}$

Cerebral oedema accounts for $57-87 \%$ of all DKA deaths. ${ }^{24} 25$ The incidence of cerebral oedema has been fairly consistent between national population based studies: $0.46 \%$ (C) (Canada), ${ }^{22} 0.68 \%$ (B) (UK), ${ }^{24}$ and $0.87 \%$ (B) (USA). ${ }^{25}$ Single centre studies often report higher frequencies because of ascertainment bias arising from secondary referral patterns: $1.1 \%$ (C) (USA) ${ }^{26}$ to $4.6 \%$ (USA). ${ }^{27}$

Reported mortality rates from cerebral oedema, in population based studies are $21 \%(\mathbf{C}),{ }^{25} 25 \%(\mathbf{C}),{ }^{22}$ and $24 \%$ (B). ${ }^{24}$ Significant morbidity is evident in $10 \%(\mathbf{C}),{ }^{22} 21 \%(\mathbf{B}),{ }^{25}$ and $26 \%(B)^{24}$ of survivors. However, some individual centres have reported markedly lower mortality and serious morbidity following DKA and cerebral oedema $\left[(\mathbf{B})(\right.$ USA $),{ }^{28} \mathbf{C}$ $\left.(\text { USA })^{29}\right]$.

Other possible causes of mortality and morbidity include hypokalaemia, hyperkalaemia, hypoglycaemia, other CNS complications; haematoma $(\mathbf{C}),{ }^{30}$ thrombosis $(\mathbf{C}),{ }^{31}$ sepsis, and infections (including rhinocerebral mucormycosis) (C), ${ }^{32}$ aspiration pneumonia, pulmonary oedema (C), ${ }^{33}$ adult respiratory distress syndrome (ARDS) (C), ${ }^{34}$ pneumomediastinum and subcutaneous emphysema (C), ${ }^{35}$ and rhabdomyolysis (C).$^{36}$ Late sequelae relate to cerebral oedema and other CNS complications; these include hypothalamopituitary insufficiency, ${ }^{37}{ }^{38}$ isolated growth hormone deficiency, ${ }^{39}$ and combined GH and TSH deficiency. ${ }^{40}$

\section{CEREBRAL OEDEMA \\ Presentation}

Cerebral oedema typically occurs 4-12 hours after treatment is activated, ${ }^{25}{ }^{41}$ but can be present before treatment has begun $\left[(\mathbf{B}){ }^{23}(\mathbf{C})^{42}{ }^{43}(\mathbf{B})^{25}\right]$, or may develop any time during treatment for DKA. Symptoms and signs of cerebral oedema are variable and include onset of headache, gradual decrease or deterioration in level of consciousness, inappropriate slowing of the pulse rate, and an increase in blood pressure (C). ${ }^{44} 45$

\section{Pathophysiology}

In vitro experiments and studies in animals and in humans presenting with cerebral oedema due to other causes (for example, trauma, stroke) suggest that the aetiopathological mechanisms may be complex. A number of mechanisms have been proposed including the role of cerebral ischaemia/ hypoxia and the generation of various inflammatory mediators, ${ }^{46}{ }^{47}$ increased cerebral blood flow, ${ }^{48}$ and disruption of cell membrane ion transport ${ }^{49} 50$ and aquaporin channels. ${ }^{51}$ The generation of intracellular organic osmolytes (myoinsoitol, taurine) and subsequent cellular osmotic imbalance has also been implicated. ${ }^{52}$ Preliminary imaging studies in children with DKA using ultrasound, computed tomography or magnetic resonance imaging indicate that some degree of cerebral oedema may be present even in patients without clinical evidence of raised intracranial pressure. ${ }^{53-56}$

\section{Demographics}

Various demographic factors have been associated with an increased risk of cerebral oedema including: presentation with new onset TIDM (B), ${ }^{23}$ (C); $;{ }^{44}$ younger age $(\mathbf{C}) ;{ }^{44}$ and longer duration of symptoms $(\mathbf{C}) .^{26}$ These associations may be a consequence of the greater likelihood of presenting with severe DKA $(\mathbf{C}){ }^{25}$

\section{Risk factors}

Several potential risk factors, at diagnosis or during treatment, have been identified through epidemiological studies.

- There is evidence that an attenuated rise in measured serum sodium concentrations during therapy for DKA may be associated with increased risk of cerebral oedema (C). ${ }^{25} 5758$ There is little evidence, however, to show associations between the volume or sodium content of intravenous fluids or rate of change in serum glucose and risk for cerebral oedema (C). ${ }^{25-275859}$ Therefore, it is unclear whether the association between sodium change and cerebral oedema reflects variations in fluid administration, or the effects of cerebral injury on renal salt handling.

- There is some evidence to support an association between severity of acidosis and risk of cerebral oedema (C).$^{60}$ There is also evidence for an association between bicarbonate treatment for correction of acidosis and increased risk of cerebral oedema (C)..$^{25} 61$

- Greater hypocapnia at presentation of DKA, after adjusting for the degree of acidosis, has been associated with cerebral oedema in two studies (C)..$^{27}$ This association correlates well with the observed detrimental effects of hypocapnia in other conditions (B). ${ }^{62}$

- Increased serum urea nitrogen at presentation of DKA is associated with increased risk of cerebral oedema (C), ${ }^{25}$ and this association may reflect greater dehydration in these patients.

Most studies show no association between the degree of hyperglycaemia at presentation of DKA with risk of cerebral oedema after correcting for other covariates (C). ${ }^{25} 27$

\section{MANAGEMENT OF DKA \\ General issues}

Children with ketosis and hyperglycaemia without vomiting or severe dehydration can be managed at home or in an outpatient health care setting (for example, emergency ward or units with similar facilities) but the level of care needs to be re-evaluated frequently and supervised by an experienced diabetes team $\left[(\mathbf{C})^{363}{ }^{64}(\mathbf{E})\right]$.

A specialist/consultant paediatrician with training and expertise in the management of DKA should direct inpatient management. The child should also be cared for in a unit which has: experienced nursing staff trained in monitoring and management; clear written guidelines; and access to laboratories for frequent evaluation of biochemical variables. 
Children with signs of severe DKA (long duration of symptoms, compromised circulation, or depressed level of consciousness) or those who are at increased risk for cerebral oedema (including $<5$ years of age, new onset) should be considered immediately for treatment in an intensive care unit (paediatric if available) or in a children's ward specialising in diabetes care with equivalent resources and supervision $\left[(\mathbf{C})^{65}(\mathbf{E})\right]$. If transfer by ambulance to another unit is required, caution should be exercised in use of sedatives and antiemetics.

\section{Monitoring}

There should be documentation of hour by hour clinical observations, intravenous and oral medication, fluids, and laboratory results during the entire treatment period $(\mathbf{E})$.

Monitoring should include the following:

- Hourly heart rate, respiratory rate, blood pressure.

- Hourly (or more frequent) accurate fluid input and output (where there is impaired level of consciousness, urinary catheterisation may be necessary).

- In severe DKA, ECG monitoring may be helpful to assess T-waves for evidence of hyperkalaemia/hypokalaemia.

- Capillary blood glucose should be monitored hourly (but must be cross checked against laboratory venous glucose as capillary methods may be inaccurate in the presence of poor peripheral circulation and acidosis).

- Laboratory tests: electrolytes, urea, haematocrit, blood glucose, and blood gases should be repeated 2-4 hourly. (However, electrolytes should be monitored hourly as clinically indicated in the more severe cases.) An increased WBC may be due to stress and cannot be taken as a sign of infection.

- Hourly or more frequent neurological observations for warning signs and symptoms of cerebral oedema:

- Headache

- Inappropriate slowing of heart rate

- Recurrence of vomiting

- Change in neurological status (restlessness, irritability, increased drowsiness, incontinence) or specific neurological signs (for example, cranial nerve palsies, pupillary response)

- Rising blood pressure

- Decreased oxygen saturation.

Those monitoring should be instructed to alert the physician of any of these manifestations, as it may be difficult to clinically discriminate cerebral oedema from other causes of altered mental status.

\section{Fluids and salt (table 1)}

The high effective osmolality of the extracellular fluid (ECF) compartment results in a shift of water from the intracellular fluid compartment (ICF) to the ECF. Studies performed in adults with TIDM in whom insulin therapy was withheld have shown fluid deficits of around 5 litres $^{66}$ together with approximately $20 \%$ loss of total body sodium and potassium. ${ }^{67}$ At the time of presentation, patients are ECF contracted and clinical estimates of the deficit are usually in the range of $7-10 \%$, although these can be subjective and may overestimate the problem. ${ }^{68}$ Shock with haemodynamic compromise is a rare event in DKA. The serum sodium measurement is an unreliable measure of the degree of ECF contraction due to the dilutional effect of fluid shift. The effective osmolality $(2[\mathrm{Na}+\mathrm{K}]+$ glucose $)$ at the time of presentation is frequently in the range of 300-350 mosm/l. Increased serum urea nitrogen and haematocrit may be useful markers of severe ECF contraction. ${ }^{28}{ }^{64}$
The onset of dehydration is associated with a reduction in glomerular filtration rate (GFR), which results in decreased glucose and ketone clearance from the blood. Studies in humans have shown that intravenous fluid administration alone results in substantial falls in blood glucose levels because of an increase in GFR. ${ }^{69}{ }^{70}$ The objectives of fluid and sodium replacement therapy in DKA are: (a) restoration of circulating volume, (b) replacement of sodium and the ECF and ICF deficit of water, (c) the restoration of GFR with enhanced clearance of glucose and ketones from the blood, and (d) the avoidance of cerebral oedema.

Both animal and human studies have shown that intracranial pressure (ICP) rises as intravenous fluids are administered. ${ }^{71}{ }^{72}$ There are also animal models of DKA which show that the use of hypotonic fluids, compared to isotonic, is associated with greater rises in ICP. ${ }^{71}$ Although there are no category A studies that show superiority of any fluid regimen over another, there are category $\mathrm{C}$ data that suggest that rapid fluid replacement with hypotonic fluid is associated with an increased risk of cerebral oedema (see section on risk factors above). There are both adult (category A) and paediatric (level B) studies which show that a less rapid fluid deficit correction with isotonic or near-isotonic solutions results in earlier reversal of acidosis. ${ }^{29}{ }^{73}$ However, the use of large amounts of $0.9 \%$ saline has also been associated with the development of hyperchloraemic metabolic acidosis. ${ }^{74} 75$

There are no data to support the use of colloids in preference to crystalloids in the treatment of DKA. There are also no data to support the use of solutions more dilute than $0.45 \% \mathrm{NaCl}$; the use of these solutions, which contain a large amount of electrolyte free water, is likely to lead to a rapid osmolar change and movement of fluid into the ICF compartment.

\section{Insulin (table 2)}

Although rehydration alone causes some decrease in blood glucose concentration, insulin therapy is essential to normalise the blood glucose concentration and to suppress lipolysis and ketogenesis. Although different routes (subcutaneous, intramuscular, intravenous) and doses have been used, extensive evidence indicates that "low dose" intravenous insulin administration should be the standard of care $^{76}$

Physiological studies indicate that intravenous insulin at a dose of 0.1 unit $/ \mathrm{kg} /$ hour, which achieves steady state plasma insulin levels of around $100-200 \mu \mathrm{U} / \mathrm{ml}$ within 60 minutes, is effective. ${ }^{77}$ Such plasma insulin levels are able to offset insulin resistance and, in most circumstances, inhibit lipolysis and ketogenesis; exerting maximal or near maximal effects on suppression of glucose production and stimulated peripheral glucose uptake. ${ }^{78}$ The resolution of acidaemia invariably takes longer than normalisation of blood glucose concentrations. ${ }^{79}$

\section{Potassium (table 3)}

Adults with DKA have total body potassium deficits of the order of 3-6 mmol/kg; data in children are sparse. ${ }^{66}{ }^{67} 82-85$ The major loss of potassium is from the intracellular pool as a result of hypertonicity, insulin deficiency, and buffering of hydrogen ions within the cell. Serum potassium levels at the time of presentation may be normal, increased or decreased: hypokalaemia at presentation may be related to prolonged duration of disease, whereas hyperkalaemia primarily results from reduced renal function. ${ }^{86}$ Administration of insulin and the correction of acidosis will drive potassium back into the cells, decreasing serum levels.

\section{Phosphate (table 3)}

Depletion of intracellular phosphate occurs and phosphate is lost as a result of osmotic diuresis. In adults, deficits are in 
Table 1 Water and salt replacement in DKA

- Water and salt deficits must be replaced. Intravenous or oral fluids that may have been given before the child presents for treatment and prior to assessment should be factored into calculation of deficit and repair. (A)

- Initial intravenous fluid administration and, if needed, volume expansion, should begin immediately with an isotonic solution $10.9 \%$ saline or balanced salt solutions such as Ringer's lactate). The volume and rate of administration depends on circulatory status and, where it is clinically indicated, the volume is typically $10-20 \mathrm{ml} / \mathrm{kg}$ over $1-2$ hours, repeated if necessary. (E)

- Use crystalloid and not colloid. (C)

- Subsequent fluid management should be with a solution with a tonicity equal to or greater than $0.45 \%$ saline. (C)

- This can be achieved by administering $0.9 \%$ saline or, balanced salt solution (Ringer's lactate or $0.45 \%$ saline with added potassium). (E) - Rate of intravenous fluid should be calculated to rehydrate evenly over at least $48 \mathrm{~h}$. (E)

- In addition to clinical assessment of dehydration, calculation of effective osmolality may be valuable to guide fluid and electrolyte therapy. (E)

- As the severity of dehydration may be difficult to determine and can be overestimated, infuse fluid each day at a rate rarely in excess of $1.5-2$ times the usual daily requirement based on age, weight, or body surface area. Urinary losses should not be added to the calculation of replacement fluids. (E)

the range $0.5-2.5 \mathrm{mmol} / \mathrm{kg}^{6}{ }^{66} 6784$ but comparable data in children are unavailable. The fall in plasma phosphate levels after starting treatment is exacerbated by insulin administration as phosphate re-enters cells.$^{87}$ Low plasma phosphate levels, when indicative of total body depletion in other conditions, have been associated with a wide array of metabolic disturbances; however, particular interest has focused on erythrocyte 2,3-diphosphoglycerate concentrations and effects on tissue oxygenation. ${ }^{88}$ Phosphate depletion persists for several days after resolution of DKA. ${ }^{62828}$ However, prospective studies have failed to show significant clinical benefit from phosphate replacement. ${ }^{99-94}$ Nevertheless, provided that careful monitoring is performed to avoid hypocalcaemia, ${ }^{95} 96$ potassium phosphate may be safely used in combination with potassium chloride or acetate to avoid hyperchloraemia.

\section{Acidosis (table 3)}

Even severe acidosis is reversible by fluid and insulin replacement. Administration of insulin stops further ketoacid synthesis and allows excess ketoacids to be metabolised. The metabolism of keto-anion results in the regeneration of bicarbonate $\left(\mathrm{HCO}_{3}\right)$ and spontaneous correction of acidaemia. Also, treatment of hypovolaemia will improve decreased tissue perfusion and renal function, thus increasing the excretion of organic acids (see "Fluids and salt" above) and reversing any lactic acidosis, which may account for $25 \%$ of the acidaemia.

In DKA there is an increased anion gap. The major retained anion is beta-hydoxybutyrate $(\beta-\mathrm{OHB})$ and acetoacetate.

Anion gap $=\left[\mathrm{Na}^{+}\right]-\left(\left[\mathrm{Cl}^{-}\right]+\left[\mathrm{HCO}_{3}{ }^{-}\right]\right)$

(normally $12 \pm 2 \mathrm{mmol} / \mathrm{l}$ )

The indications for bicarbonate therapy in DKA are unclear. Several controlled trials of sodium bicarbonate in small numbers of children and adults ((B), (C)) have been unable to show clinical benefit or any important difference in the rate of rise in the plasma bicarbonate concentration (C). ${ }^{25}$ 97-100

There are potential arguments against the use of bicarbonate. ${ }^{25} 97101102$ Of concern is that bicarbonate therapy may cause paradoxical CNS acidosis and that rapid correction of acidosis caused by bicarbonate will result in hypokalaemia, may accentuate sodium load, and contribute to serum hypertonicity. In addition, alkali therapy may increase hepatic ketone production, thus slowing the rate of recovery from the ketosis.

These findings, however, do not address the issue that there may be selected patients who may benefit from cautious alkali therapy. These include patients with severe acidaemia (arterial pH <6.9) in whom decreased cardiac contractility and peripheral vasodilatation can further impair tissue perfusion, and patients with potentially life threatening hyperkalaemia.

\section{Treatment of cerebral oedema}

Treatment should be initiated as soon as the condition is suspected. The rate of fluid administration should be reduced. Although mannitol has been shown to have possible beneficial effects in case reports, ${ }^{103-105}$ there has been no definite beneficial or detrimental effect in retrospective epidemiological studies. ${ }^{106}$ The response may be altered by timing of administration, delayed administration being less effective. Intravenous mannitol should be given (0.25-1.0 g/ $\mathrm{kg}$ over $20 \mathrm{~min}$ ) in patients with signs of cerebral oedema before impending respiratory failure (C), (E). Repeat in two hours if there is no initial response. Hypertonic saline $(3 \%)$ $5-10 \mathrm{ml} / \mathrm{kg}$ over $30 \mathrm{~min}$ may be an alternative to mannitol (C). ${ }^{107}$

Intubation and ventilation may be necessary. However, aggressive hyperventilation has been associated with poor outcome in one retrospective study of DKA related cerebral oedema; ${ }^{106}$ similarly detrimental effects have been reported in numerous other conditions such as head trauma and high

Table 2 Insulin therapy for DKA

- Correction of insulin deficiency: (A)

- Dose: 0.1 units $/ \mathrm{kg} /$ hour (A)

- Route of administration: intravenous (A)

- The dose of insulin should remain at $0.1 \mathrm{U} / \mathrm{kg} /$ hour at least until resolution of ketoacidosis $\left(\mathrm{pH}>7.30, \mathrm{HCO}_{3}>15 \mathrm{mmol} / \mathrm{l}\right.$ and/or closure of anion gap). To prevent an unduly rapid decrease in plasma glucose concentration and possible development of hypoglycemia, glucose should be added to the intravenous fluid when the plasma glucose falls to $\sim 14-17 \mathrm{mmol} / \mathrm{l}(250-300 \mathrm{mg} / \mathrm{dl})$. (B)

- There may be circumstances where the insulin dose may be safely reduced earlier, but the criteria have not been defined. (E)

- If biochemical parameters of ketoacidosis ( $\mathrm{pH}$, anion gap) do not improve, reassess the patient, review insulin therapy, and consider other possible causes of impaired response to insulin; e.g. infection, errors in insulin preparation, adhesion of insulin to tubing with very dilute solutions. (E)

- There is evidence that an intravenous bolus of insulin is not necessary (C). ${ }^{80} 81$ However, a bolus may be used at the start of insulin therapy, particularly if insulin treatment has been delayed. (E)

- In unusual circumstances where intravenous administration is not possible, the intramuscular or subcutaneous route of insulin administration has been used effectively (C). ${ }^{76}$ However, poor perfusion will impair absorption of insulin. ${ }^{79}$ 
Table 3 Potassium, phosphate, and acid base management

Potassium

- Replacement is required. (A)

- Replacement therapy should be based on serum potassium measurements. (E)

- Start potassium replacement immediately if the patient is hypokalaemic; otherwise, start potassium concurrent with starting insulin therapy. If the patient is hyperkalaemic, defer potassium until urine output is documented. (E)

- Starting potassium concentration in the infusate should be $40 \mathrm{mmol} / \mathrm{I}$ (E) and potassium replacement should continue throughout intravenous fluid therapy. (E)

Phosphate

- There is no evidence that replacement has clinical benefit (A). Severe hypophosphataemia should be treated. (C)

- Potassium phosphate salts may be used as an alternative to or combined with potassium chloride/acetate. (C)

- Administration of phosphate may induce hypocalcaemia. (C)

Acid base

- Other acute resuscitation protocols no longer recommend bicarbonate administration unless the acidosis is "profound" and "likely to affect the action of adrenaline/epinephrine during resuscitation". (A)

- Fluid and insulin replacement without bicarbonate administration corrects ketoacidosis. (A)

- Data show that treatment with bicarbonate confers no clinical benefit. (B)

- Repair fluids containing various buffering agents (bicarbonate acetate, lactate) have been used (C). The efficacy and safety of these agents have not been established.

altitude exposure. ${ }^{62}$ There are no data regarding glucocorticoid use in DKA related cerebral oedema.

\section{PREVENTION OF DKA}

\section{Before diagnosis}

Earlier diagnosis through genetic and immunological screening of high risk children such as in the recent DPT-1 study ${ }^{108}$ decrease DKA incidence at diabetes onset (A). ${ }^{14} 109$ High levels of awareness related to the existence of other members of families with TIDM also reduces risk of DKA. A school and physician awareness campaign, targeted at 6-14 year olds, reduced rates of DKA from $78 \%$ to almost $0 \%$ over a six year period. (B). ${ }^{110}$ Increased public awareness of signs and symptoms of diabetes should lead to earlier diagnosis, particularly in children under the age of 5 years where checking urine or blood for glucose may prevent misdiagnosis (E). Although such strategies are intuitively obvious, programmes to decrease DKA at onset need to be designed and evaluated in diverse populations and age groups.

\section{Beyond diagnosis}

Studies of the effects of comprehensive diabetes programmes and telephone helplines report a reduction in the rates of DKA from 15-60 to 5-5.9/100 patient-years (B). ${ }^{1111} 112$ In patients on continuous subcutaneous insulin pumps, episodes of DKA can be reduced with the introduction of educational algorithms (E). Therefore, it is likely that episodes of DKA after diagnosis could be reduced if all children with diabetes receive comprehensive diabetes health care and education, and have access to a 24 hour diabetes telephone helpline (B). ${ }^{19}$ The value of home measurement of betahydroxybutyrate as a mechanism for earlier diagnosis and thus prevention of hospitalisation needs to be assessed.

Multiple episodes of recurrent DKA are more problematic: in a recent UK study $4.8 \%$ of patients accounted for $22.5 \%$ of all episodes over a three year period. ${ }^{24}$ Insulin omission has been identified as the major factor in most of these cases and may be confirmed by finding low free insulin levels on admission (C).$^{13113}$ There is no evidence that mental health interventions alone can impact on the frequency of DKA in these children (B), ${ }^{141711}$ but insulin omission can be prevented by sequential schemes providing education, psychosocial evaluation, and treatment combined with adult supervision of insulin administration (B) ${ }^{17}$ When responsible adults administer insulin, a tenfold reduction in episodes of DKA has been reported (B). ${ }^{17}$

\section{KEY ISSUES FOR FUTURE INVESTIGATION Prevention}

- Efficacy and cost effectiveness of strategies to reduce DKA incidence. Frequency and evaluation of ketoacidosis in type 2 diabetes mellitus.

\section{Management}

- Improved assessment of dehydration. Systematic evaluation of rehydration solutions, such as those containing bicarbonate, acetate, lactate, and phosphate. Use of lower doses of insulin in younger children. Criteria for reducing dose of insulin during treatment of DKA need to be clarified. Need for bicarbonate therapy in those with $\mathrm{pH}$ $<6.9$ and the very young.

\section{Cerebral oedema}

- Meta-analysis of existing epidemiological studies to identify factors related to increased risk in infants, and the newly diagnosed. Monitoring of DKA and earlier detection of signs of cerebral oedema. Efficacy of hypertonic saline versus mannitol.

\section{ACKNOWLEDGEMENTS}

The LWPES and ESPE gratefully acknowledge Aventis Pharmaceuticals, Novo Nordisk, and The Society for Endocrinology for their support of the consensus conference, Sten Renstad of Semser for organising the conference, and Emily Knight and Kathy Wypychowski for assistance with preparing the consensus statement.

\section{Authors' affiliations}

D B Dunger, C L Acerini, T P A Danne, R Hanas, M O Savage,

R C Tasker, European Society for Paediatric Endocrinology (ESPE) M A Sperling, D J Bohn, D Daneman, N S Glaser, R L Hintz, L L Levitsky, J I Wolfsdorf, Lawson Wilkins Pediatric Endocrine Society (LWPES)

Participants: Carlo L Acerini, Cambridge UK; Dorothy J Becker, Pittsburgh, USA; Desmond Bohn, Toronto, Canada; Stuart J Brink, Waltham, USA; Francesco Chiarelli, Chieti, Italy; Maria Craig, Kogarth, Australia; Gisela Dahlquist, Umea, Sweden; Denis Daneman, Toronto, Canada; Thomas Danne, Hanover, Germany; David B Dunger, Cambridge, UK; Julie A Edge, Oxford, UK; Irma Fiordalisi, Greenville, USA; Nicole S Glaser, Sacramento, USA; John Gregory, Cardiff, UK; Mitchell Halperin, Toronto, Canada; Ragnar Hanas, Uddevalla, Sweden; Glen Harris, Greenville, USA; Morey W Haymond, Houston, USA; Ray L Hintz, Stanford, USA; Carol Inward, Cardiff, UK; Chris Kelnar, Edinburgh, UK; Wieland Kiess, Leipzig, Germany; Mikael Knip, Helinski, Finland; Elliot J Krane, Stanford, USA; Nathan Kuppermann, Sacramento, USA; Sarah Muirhead Lawrence, Ottawa, Canada; Lynne Levitsky, Boston, USA; Marc Maes, Brussels, Belgium; Henrik Mortensen, Glostrup, Denmark; Andrew Muir, Augusta, USA; Andreas Neu, Tubingen, Germany; Jose Ramet, Brussels, Belgium; Robert Rapaport, New York, USA; Arleta Rewers, Denver, USA; Marian J Rewers, Denver, USA; Arlan L Rosenbloom, Gainesville, USA; Martin O Savage, London, UK; Mark A Sperling, Pittsburgh, USA; Peter Swift, Leicester, UK; William V Tamborlane, New Haven, USA; Robert C Tasker, Cambridge, UK; Nadia Tubiana-Rufi, Paris, France; Maurizio Vanelli, Parma, Italy; Diane K Wherrett, Toronto, Canada; Neil H White, St Lovis, USA; Joseph I Wolfsdorf, Boston, USA.

\section{APPENDIX}

Table Al presents the ADA evidence grading system for clinical practice recommendations. 
Table A1 ADA evidence grading system for clinical practice recommendations*

Level of
evidence

A

Description

Clear evidence from well conducted, generalisable, randomised controlled trials that are adequately powered, including:

- Multicentre trial

- Meta-analysis incorporating quality ratings

- Compelling non-experimental evidence (i.e. "all or none" rule) developed by the Centre for Evidence Based Medicine at Oxfordt

Supportive evidence from well conducted randomised controlled trials that are adequately powered, including:

- Well conducted trials at one or more institutions

B Supportive evidence from well conducted cohort studies, including:

- Prospective cohort studies or registry

- Meta-analysis of cohort studies Supportive evidence from a well conducted case-control study

C Supportive evidence from poorly controlled or uncontrolled studies including:

- Randomised clinical trials with one or more major or three or more minor methodological flaws that could invalidate the results

- Observational studies with high potential for bias

- Case series or case reports

Conflicting evidence with the weight of evidence supporting the recommendation

E Expert consensus or clinical experience

*Diabetes Care 2003;26(suppl 1):S1.

†Either all patients died before therapy and at least some survived with therapy or some patients died without therapy and none died with

therapy. Example: use of insulin in the treatment of diabetes ketoacidosis.

\section{REFERENCES}

1 ADA. American Diabetes Association Position Statement: Hyperglycemic crises in patients with diabetes mellitus. Diabetes Care 2003;26(suppl 1):S1-S2

2 ISPAD. Consensus guidelines. www.ispad.org, 2000.

3 Chase HP, Garg SK, Jelley DH. Diabetic ketoacidosis in children and the role of outpatient management. Pediatr Rev 1990;1 1:297-304.

4 Pinkey JH, et al. Presentation and progress of childhood diabetes mellitus: a prospective population-based study. The Bart's-Oxford Study Group. Diabetologia 1994;37:70-4.

5 Levy-Marchal C, et al. Clinical and laboratory features of type 1 diabetic children at the time of diagnosis. Diabet Med 1992;9:279-84.

6 Komulainen J, et al. Ketoacidosis at the diagnosis of type 1 (insulin dependent) diabetes mellitus is related to poor residual beta cell function. Childhood Diabetes in Finland Study Group. Arch Dis Child 1996;75:410-15.

7 Curtis JR, et al. Recent trends in hospitalization for diabetic ketoacidosis in Ontario children. Diabetes Care 2002;25:1591-6.

8 Hirasing RA, et al. Trends in hospital admissions among children aged 0-19 years with type I diabetes in the Netherlands. Diabetes Care 1996;19:431-4.

9 Komulainen J, et al. Clinical, autoimmune, and genetic characteristics of very young children with type 1 diabetes. Childhood Diabetes in Finland (DiMe) Study Group. Diabetes Care 1999;22:1950-5.

10 Alavi IA, Sharma BK, Pillay VK. Steroid-induced diabetic ketoacidosis. Am J Med Sci 1971;262:15-23.

11 Goldstein LE, et al. New-onset diabetes mellitus and diabetic ketoacidosis associated with olanzapine treatment. Psychosomatics 1999;40:438-43.

12 Smith CP, et al. Ketoacidosis occurring in newly diagnosed and established diabetic children. Acta Paediatr 1998;87:537-41.

13 Morris AD, et al. Adherence to insulin treatment, glycaemic control, and ketoacidosis in insulin-dependent diabetes mellitus. The DARTS/MEMO Collaboration. Diabetes Audit and Research in Tayside Scotland. Medicines Monitoring Unit. Lancet 1997;350:1505-10.

14 Rewers A, et al. Predictors of acute complications in children with type 1 diabetes. JAMA 2002;287:2511-18.

15 Rosilio $M$, et al. Factors associated with glycemic control. A cross-sectional nationwide study in 2,579 French children with type 1 diabetes. The French Pediatric Diabetes Group. Diabetes Care 1998;21:1146-53.

16 Keenan HT, Foster CM, Bratton SL. Social factors associated with prolonged hospitalization among diabetic children. Pediatrics 2002;109:40-4.

17 Golden MP, Herrold AJ, Orr DP. An approach to prevention of recurrent diabetic ketoacidosis in the pediatric population. J Pediatr 1985; 107:195-200

18 Flood RG, Chiang VW. Rate and prediction of infection in children with diabetic ketoacidosis. Am J Emerg Med 2001;19:270-3.
19 Hoffman WH, et al. Service and education for the insulin-dependent child. Diabetes Care 1978;1:285-8.

20 Glasgow AM, et al. Readmissions of children with diabetes mellitus to a children's hospital. Pediatrics 1991;88:98-104.

21 Levitsky L, et al. Death from diabetes (DM) in hospitalized children (19701988). Pediatr Res 1991;29:A195 (abstract).

22 Cummings $E$, Lawrence S, Daneman D. Cerebral edema (CE) in pediatric diabetic ketoacidosis (DKA) in Canada. Diabetes 2003:52:A400 (abstract).

23 Edge JA, Ford-Adams ME, Dunger DB. Causes of death in children with insulin dependent diabetes 1990-96. Arch Dis Child 1999;81:318-23.

24 Edge JA, et al. The risk and outcome of cerebral oedema developing during diabetic ketoacidosis. Arch Dis Child 2001:85:16-22.

25 Glaser N, et al. Risk factors for cerebral edema in children with diabetic ketoacidosis. The Pediatric Emergency Medicine Collaborative Research Committee of the American Academy of Pediatrics. N Engl J Med $2001 ; 344: 264-9$

26 Bello FA, Sotos JF. Cerebral oedema in diabetic ketoacidosis in children. Lancet 1990;336:64.

27 Mahoney CP, Vlcek BW, DelAguila M. Risk factors for developing brain herniation during diabetic ketoacidosis. Pediatr Neurol 1999;21:721-7.

28 Harris GD, Fiordalisi I. Physiologic management of diabetic ketoacidemia. A 5 -year prospective pediatric experience in 231 episodes. Arch Pediatr Adolesc Med 1994;148:1046-52.

29 Felner El, White PC. Improving management of diabetic ketoacidosis in children. Pediatrics 2001;108:735-40.

30 Atluru VL. Spontaneous intracerebral hematomas in juvenile diabetic ketoacidosis. Pediatr Neurol 1986;2:167-9.

31 Kanter RK, et al. Arterial thrombosis causing cerebral edema in association with diabetic ketoacidosis. Crit Care Med 1987;15:175-6.

32 Moll GW Jr, et al. Rhinocerebral mucormycosis in IDDM. Sequential magnetic resonance imaging of long-term survival with intensive therapy. Diabetes Care 1994; 17:1348-53.

33 Young MC. Simultaneous acute cerebral and pulmonary edema complicating diabetic ketoacidosis. Diabetes Care 1995:18:1288-90.

34 Breidbart $\mathbf{S}$, et al. Adult respiratory distress syndrome in an adolescent with diabetic ketoacidosis. J Pediatr 1987;111:736-8.

35 Watson JP, Barnett AH. Pneumomediastinum in diabetic ketoacidosis. Diabet Med 1989:6:173-4.

36 Cateels K, et al. Rhabdomyolysis in diabetic ketoacidosis. Pediatric Diabetes 2003:4:29-31.

37 Tubiana-Rufi N, Thizon-de Gaulle I, Czernichow P. Hypothalamopituitary deficiency and precocious puberty following hyperhydration in diabetic ketoacidosis. Horm Res 1992:37:60-3.

38 Lufkin EG, et al. Acute cerebral dysfunction in diabetic ketoacidosis: survival followed by panhypopituitarism. Metabolism 1977;26:363-9.

39 Keller RJ, Wolfsdorf Jl. Isolated growth hormone deficiency after cerebral edema complicating diabetic ketoacidosis. N Engl J Med 1987;316:857-9.

40 Dunlop KA, Woodman D, Carson DJ. Hypopituitarism following cerebral oedema with diabetic ketoacidosis. Arch Dis Child 2002;87:337-8.

41 Edge JA. Cerebral oedema during treatment of diabetic ketoacidosis: are we any nearer finding a cause? Diabetes Metab Res Rev 2000;16:316-24.

42 Deeb L. Development of fatal cerebral oedema during outpatient therapy for diabetic ketoacidosis. Pract Diab 1989;6:212-13

43 Glasgow AM. Devastating cerebral edema in diabetic ketoacidosis before therapy. Diabetes Care 1991:14:77-8.

44 Rosenbloom AL. Intracerebral crises during treatment of diabetic ketoacidosis. Diabetes Care 1990;13:22-33.

45 Muir A. Therapeutic controversy. Cerebral edema in diabetic ketoacidosis: a look beyond rehydration. J Clin Endocrinol Metab 2000;85:509-13.

46 Abbott NJ. Inflammatory mediators and modulation of blood-brain barrier permeability. Cell Mol Neurobiol 2000;20:131-47.

47 Sarker MH, Fraser PA. The role of guanylyl cyclases in the permeability response to inflammatory mediators in pial venular capillaries in the rat. J Physiol 2002;540(pt 1):209-18.

48 Yang GY, Betz AL. Reperfusion-induced injury to the blood-brain barrier after midd́le cerebral artery occlusion in rats. Stroke 1994;25:1658-65.

49 O'Donnell ME, Martinez A, Sun D. Endothelial Na-K-Cl cotransport regulation by tonicity and hormones: phosphorylation of cotransport protein Am J Physiol 1995;269(6 pt 1):C1513-23.

50 Kimelberg HK, et al. Astrocytic swelling due to hypotonic or high $\mathrm{K}+$ medium causes inhibition of glutamate and aspartate uptake and increases their release. J Cereb Blood Flow Metab 1995;15:409-16.

51 Manley GT, et al. Aquaporin-4 deletion in mice reduces brain edema after acute water intoxication and ischemic stroke. Nat Med 2000;6:159-63.

52 McManus ML, Churchwell KB, Strange K. Regulation of cell volume in health and disease. N Engl J Med 1995;333:1260-6.

53 Krane EJ, et al. Subclinical brain swelling in children during treatment of diabetic ketoacidosis. N Engl J Med 1985;312:1147-51.

54 Hoffman WH, et al. Cranial CT in children and adolescents with diabetic ketoacidosis. AJNR Am J Neuroradiol 1988:9:733-9.

55 Hoffman WH, et al. Computer analysis of magnetic resonance imaging of the brain in children and adolescents after treatment of diabetic ketoacidosis. J Diabetes Complications 1999;13:176-81.

56 Hoffman WH, et al. Transcranial Doppler ultrasound assessment of intracranial hemodynamics in children with diabetic ketoacidosis. I Clin Ultrasound 1995;23:517-23.

57 Harris GD, et al. Minimizing the risk of brain herniation during treatment of diabetic ketoacidemia: a retrospective and prospective study [published erratum appears in J Pediatr 1991;118:166-7]. J Pediatr 1990;117(1 pt 1):22-31. 
58 Hale PM, et al. Factors predicting cerebral edema in young children with diabetic ketoacidosis and new onset type I diabetes. Acta Paediatr 1997;86:626-31.

59 Mel JM, Werther GA. Incidence and outcome of diabetic cerebral oedema in childhood: are there predictors? J Paediatr Child Health 1995;31:17-20.

60 Durr JA, et al. Correlates of brain edema in uncontrolled IDDM. Diabetes 1992;41:627-32.

61 Bureau MA, et al. Cerebral hypoxia from bicarbonate infusion in diabetic acidosis. J Pediatr 1980;96:968-73.

62 Roberts I, Shhierhout G. Hyperventilation therapy for acute traumatic brain injury (Cochrane review). The Cochrane Library 2003.

63 Bonadio WA, et al. Outpatient management of diabetic ketoacidosis. Am J Dis Child 1988;142:448-50.

64 Linares MY, Schunk JE, Lindsay R. Laboratory presentation in diabetic ketoacidosis and duration of therapy. Pediatr Emerg Care 1996;12:347-51.

65 Monroe KW, King W, Atchison JA. Use of PRISM scores in triage of pediatric patients with diabetic ketoacidosis. Am J Manag Care 1997;3:253-8.

66 Atchley D, Loeb R, Richards D. On diabetic acidosis. A detailed study of electrolyte balances following withdrawal and reestablishment of therapy. J Clin Invest 1933;12:297-326.

67 Nabarro JDN, Spencer AG, Stowers JM. Metabolic studies in severe diabetic ketosis. Q J Med 1952;82:225-48.

68 Mackenzie A, Barnes G, Shann F. Clinical signs of dehydration in children. Lancet 1989;2:605-7.

69 Waldhausl W, et al. Severe hyperglycemia: effects of rehydration on endocrine derangements and blood glucose concentration. Diabetes 1979;28:577-84.

70 Owen OE, Licht JH, Sapir DG. Renal function and effects of partial rehydration during diabetic ketoacidosis. Diabetes 1981;30:510-18.

71 Harris GD, Fiordalisi I, Yu C. Maintaining normal intracranial pressure in a rabbit model during treatment of severe diabetic ketoacidemia. Life Sci 1996:59:1695-702.

72 Clements RS Jr, et al. Increased cerebrospinal-fluid pressure during treatment of diabetic ketosis. Lancet 1971;2:671-5.

73 Adrogue HJ, Barrero J, Eknoyan G. Salutary effects of modest fluid replacement in the treatment of adults with diabetic ketoacidosis. Use in patients without extreme volume deficit. JAMA 1989;262:2108-13.

74 Adrogue HJ, Eknoyan G, Suki WK. Diabetic ketoacidosis: role of the kidney in the acid-base homeostasis re-evaluated. Kidney Int 1984;25:591-8.

75 Oh MS, Carroll HJ, Uribarri J. Mechanism of normochloremic and hyperchloremic acidosis in diabetic ketoacidosis. Nephron 1990;54:1-6.

76 Kitabchi AE. Low-dose insulin therapy in diabetic ketoacidosis: fact or fiction? Diabetes Metab Rev 1989;5:337-63.

77 Schade DS, Eaton RP. Dose response to insulin in man: differential effects on glucose and ketone body regulation. J Clin Endocrinol Metab 1977:44:1038-53.

78 Luzi L, et al. Metabolic effects of low-dose insulin therapy on glucose metabolism in diabetic ketoacidosis. Diabetes 1988;37:1470-7.

79 Soler NG, et al. Comparative study of different insulin regimens in management of diabetic ketoacidosis. Lancet 1975;2:1221-4.

80 Fort $\mathbf{P}$, Waters SM, Lifshitz F. Low-dose insulin infusion in the treatment of diabetic ketoacidosis: bolus versus no bolus. J Pediatr 1980;96:36-40.

81 Lindsay R, Bolte RG. The use of an insulin bolus in low-dose insulin infusion for pediatric diabetic ketoacidosis. Pediatr Emerg Care 1989:5:77-9.

82 Nabarro J, Spencer A, Stowers J. Treatment of diabetic ketoacidosis. Lancet 1952;i:983.

83 Danowski T, Peters J. Studies in diabetic acidosis and coma, with particular emphasis on the retention of administered potassium. J Clin Invest 1949;28: 1-9.

84 Butler A, Talbot N. Metabolic studies in diabetic coma. Trans Assoc Am Physicians 1947;60:102-9.

85 Darrow DC, Pratt EL. Retention of water and electrolyte during recovery in a patient with diabetic acidosis. J Pediatr 1952;41:688-96.

86 Adrogue $\mathrm{HJ}$, et al. Determinants of plasma potassium levels in diabetic ketoacidosis. Medicine (Baltimore) 1986;65:163-72

87 Riley MS, Schade DS, Eaton RP. Effects of insulin infusion on plasma phosphate in diabetic patients. Metabolism 1979;28:191-4.
88 Alberti KG, et al. 2,3-Diphosphoglycerate and tissue oxygenation in uncontrolled diabetes mellitus. Lancet 1972;2:391-5.

89 Gibby OM, et al. Oxygen availability from the blood and the effect of phosphate replacement on erythrocyte 2,3-diphosphoglycerate and haemoglobin-oxygen affinity in diabetic ketoacidosis. Diabetologia 1978; 15:381-5.

90 Keller U, Berger W. Prevention of hypophosphatemia by phosphate infusion during treatment of diabetic ketoacidosis and hyperosmolar coma. Diabetes 1980;29:87-95

91 Wilson HK, et al. Phosphate therapy in diabetic ketoacidosis. Arch Intern Med 1982;142:517-20.

92 Becker DJ, et al. Phosphate replacement during treatment of diabetic ketosis. Effects on calcium and phosphorus homeostasis. Am J Dis Child 1983; 137:241-6.

93 Fisher JN, Kitabchi AE. A randomized study of phosphate therapy in the treatment of diabetic ketoacidosis. J Clin Endocrinol Metab 1983:57: 177-80

94 Clerbaux T, et al. Effect of phosphate on oxygen-hemoglobin affinity, diphosphoglycerate and blood gases during recovery from diabetic ketoacidosis. Intensive Care Med 1989;15:495-8.

95 Winter RJ, et al. Diabetic ketoacidosis. Induction of hypocalcemia and hypomagnesemia by phosphate therapy. Am J Med 1979;67:897-900.

96 Zipf WB, et al. Hypocalcemia, hypomagnesemia, and transient hypoparathyroidism during therapy with potassium phosphate in diabetic ketoacidosis. Diabetes Care 1979;2:265-8.

97 Soler NG, et al. Potassium balance during treatment of diabetic ketoacidosis with special reference to the use of bicarbonate. Lancet 1972;2:665-7.

98 Hale PJ, Crase J, Nattrass M. Metabolic effects of bicarbonate in the treatment of diabetic ketoacidosis. BMJ 1984;289:1035-8.

99 Okuda $Y$, et al. Counterproductive effects of sodium bicarbonate in diabetic ketoacidosis. J Clin Endocrinol Metab 1996;81:314-20.

100 Green SM, et al. Failure of adjunctive bicarbonate to improve outcome in severe pediatric diabetic ketoacidosis. Ann Emerg Med 1998:31:41-8.

101 Ohman JL Jr, et al. The cerebrospinal fluid in diabetic ketoacidosis. N Engl J Med 1971;284:283-90.

102 Assal JP, et al. Metabolic effects of sodium bicarbonate in management of diabetic ketoacidosis. Diabetes 1974;23:405-11

103 Franklin B, Liu J, Ginsberg-Fellner F. Cerebral edema and ophthalmoplegia reversed by mannitol in a new case of insulin-dependent diabetes mellitus. Pediatrics 1982;69:87-90.

104 Shabbir N, et al. Recovery from symptomatic brain swelling in diabetic ketoacidosis. Clin Pediatr (Phila) 1992:31:570-3.

105 Roberts MD, Slover R, Chase HP. Diabetic ketoacidosis with intracerebral complications. Pediatr Diabetes 2001;2:109-14.

106 Marcin JP, et al. Factors associated with adverse outcomes in children with diabetic ketoacidosis-related cerebral edema. J Pediatr 2002:141:793-7.

107 Kamat $\mathbf{P}$, et al. Use of hypertonic saline for the treatment of altered mental status associated with diabetic ketoacidosis. Pediatr Crit Care Med 2003;4:239-42

108 Diabetes Prevention Trial - Type 1 Diabetes Study Group. Effects of insulin in relatives of patients with type 1 diabetes mellitus. N Engl J Med 2002;346: 1685-91

109 Levy-Marchal C, Patterson CC, Green A. Geographical variation of presentation at diagnosis of type I diabetes in children: the EURODIAB study. European and Diabetes. Diabetologia 2001:44(suppl 3):B75-80.

110 Vanelli $M$, et al. Effectiveness of a prevention program for diabetic ketoacidosis in children. An 8-year study in schools and private practices. Diabetes Care 1999;22:7-9.

111 Grey M, et al. Coping skills training for youth with diabetes mellitus has longlasting effects on metabolic control and quality of life. J Pediatr 2000;137:107-13.

112 Drozda DJ, et al. Assessment of the effect of a comprehensive diabetes management program on hospital admission rates of children with diabetes mellitus. Diabetes Educ 1990;16:389-93.

113 Malone Jl, Root AW. Plasma free insulin concentrations: keystone to effective management of diabetes mellitus in children. J Pediatr 1981;99:862-7. 